\title{
DOIS NOVOS ALCALOIDES AZAFENANTRENO DE Anaxagorea dolichocarpa Sprague \& Sandwith
}

\author{
Kaio A. Sales ${ }^{\mathrm{a}}$, Anderson A. V. Pinheiro ${ }^{\mathrm{a}}$, Diego I. A. F. Araújo ${ }^{\mathrm{a}}$, Rodrigo S. de Andrade ${ }^{\mathrm{a}}$, Maria de Fátima Agra ${ }^{\mathrm{b}}$, Marianna \\ V. Sobrala ${ }^{a}$ Hemerson I. F. Magalhães a, Valgrícia M. de Sousa ${ }^{a}$, Raimundo Braz-Filhoc, Marcelo S. da Silva ${ }^{\text {a }}$ e Josean F. \\ Tavares $^{\mathrm{a}, *,(1)}$ \\ ${ }^{a}$ Centro de Ciências da Saúde, Universidade Federal da Paraíba, 58051-900 João Pessoa - PB, Brasil \\ ${ }^{b}$ Departamento de Biotecnologia, Universidade Federal da Paraíba, 58051-900 João Pessoa - PB, Brasil \\ 'Departamento de Química, Instituto de Química, Universidade Federal Rural do Rio de Janeiro, 23890-000 Seropédica - RJ, Brasil
}

Recebido em 01/11/2019; aceito em 09/03/2020; publicado na web em 20/04/2020

\begin{abstract}
TWO NEW AZAPHENANTHRENE ALKALOIDS FROM Anaxagorea dolichocarpa Sprague \& Sandwith. A chemical investigation of Anaxagorea dolichocarpa Sprague \& Sandwith, a member of Annonaceae family, was carried out. The ethanolic extract from the roots of this plant led, by chromatography tecniches, to isolation of the new azaphenanthrene alkaloids dolichocarpine (1) and 9-methoxyeupolauramine (2), besides the known alkaloids eupolauramine (3), 3-methoxyeupolauridine (4), eupolauramine (5) and 4-methylsampangine (6). The structures of isolated compounds were established by 1D and 2D NMR, HRESIMS, tandem MS $^{\mathrm{n}}$ and IR data. The cytotoxicity of compounds $\mathbf{1}-\mathbf{5}$ was evaluated against HCT-116 (human colorectal carcinoma) and L929 (murine fibroblast) cell lines.
\end{abstract}

Keywords: Annonaceae; Anaxagorea; alkaloid; cytotoxicity.

\section{INTRODUÇÃO}

Annonaceae é uma família de árvores floríferas, arbustos e cipós com distribuição pantropical, sendo composta por 110 gêneros e aproximadamente 2.430 espécies. ${ }^{1,2}$ No Brasil apresenta ampla distribuição, com cerca de 390 espécies encontradas principalmente na Região Amazônica e Mata Atlântica. ${ }^{3,4}$ As espécies da família são conhecidas pela biossíntese de alcaloides e os estudos demonstraram considerável diversidade estrutural, além de notáveis atividades biológicas para esses metabólitos secundários. ${ }^{5,6}$ Com relação ao gênero, Anaxagorea compreende 26 espécies, ${ }^{7}$ ocorrendo nas regiões neotropical e paleotropical, e no Brasil é encontrado principalmente no Norte e Nordeste. ${ }^{8-10}$ Das espécies do gênero investigadas até o presente momento, foram identificados principalmente alcaloides, terpenoides, xantonas, flavonoides e lignoides. ${ }^{11-16}$ Ao passo que em Anaxagorea dolichocarpa Sprague \& Sandwith, estudos fitoquímicos prévios permitiram o isolamento de alcaloides azafenantrenos e aporfínicos..$^{11,17}$ Diversos compostos dessas duas subclasses de alcaloides já foram testados em ensaios de atividade citotóxica e antitumoral, alguns deles apresentando resultados positivos, sendo por isso considerados promissores. ${ }^{5,11}$

Historicamente, produtos naturais obtidos de plantas representam a principal fonte de novos fármacos, além de fornecerem protótipos para a síntese de compostos farmacologicamente ativos, particularmente com atividade anticâncer. Considerando que o câncer é uma das doenças com as mais altas taxas de morbidade e mortalidade no mundo, há um considerável interesse científico e comercial na descoberta de novas drogas a partir de fontes naturais. ${ }^{18}$ Então, como continuidade dos estudos do nosso grupo de pesquisa com a família Annonaceae, este trabalho buscou isolar e identificar novos compostos de A. dolichocarpa por meio de métodos cromatográficos e espectroscópicos, respectivamente, e ainda avaliar a citotoxicidade dessas substâncias.

\footnotetext{
*e-mail: josean@ltf.ufpb.br
}

\section{RESULTADOS E DISCUSSÃO}

O estudo químico do extrato etanólico das raízes de A. dolichocarpa levou ao isolamento de seis compostos (1 - 6) (Figura 1), sendo esse o primeiro relato das substâncias $\mathbf{1}$ e $\mathbf{2}$ na literatura. Suas estruturas químicas foram determinadas com base na análise dos dados espectroscópicos de RMN uni e bidimensionais, espectrometria de massa de alta resolução com ionização por electrospray (EMAR-IES) e comparação com dados da literatura. Os dados de RMN de ${ }^{1} \mathrm{H} \mathrm{e}{ }^{13} \mathrm{C}$ dos compostos 1 e $\mathbf{2}$ estão compilados na Tabela 1.

$\mathrm{O}$ composto 1 foi isolado como cristais amarelados. $\mathrm{O}$ espectro de EMAR-IES mostrou o pico do íon $[\mathrm{M}+\mathrm{H}]^{+} \operatorname{com~} \mathrm{m} / \mathrm{z}, 283,1073$ (calcd. para $\mathrm{C}_{16} \mathrm{H}_{15} \mathrm{~N}_{2} \mathrm{O}_{3}, 283,1077$ ), compatível com a fórmula molecular $\mathrm{C}_{16} \mathrm{H}_{15} \mathrm{~N}_{2} \mathrm{O}_{3}$. O espectro de infravermelho mostrou absorção de duas bandas em 3306 e $3139 \mathrm{~cm}^{-1}$ de estiramento $\mathrm{N}-\mathrm{H}_{2}$, banda de carbonila de amida em $1646 \mathrm{~cm}^{-1}$, absorções em 1618 e $1582 \mathrm{~cm}^{-1} \mathrm{de}$ $\mathrm{C}=\mathrm{C}$ de aromático, além do sinal em $1055 \mathrm{~cm}^{-1}$ de estiramento $\mathrm{C}-\mathrm{O}$. O espectro de $\mathrm{RMN}$ de ${ }^{1} \mathrm{H}\left(\mathrm{CD}_{3} \mathrm{OD}, 400 \mathrm{MHz}\right)$ mostrou seis sinais de prótons aromáticos, sendo dois dupletos em $\delta_{\mathrm{H}} 8,95(\mathrm{~d} ; J=4,8 \mathrm{~Hz})$ e $\delta_{\mathrm{H}} 7,64(\mathrm{~d} ; J=4,8 \mathrm{~Hz})$, sugestivos para os prótons $\mathrm{H}-2$ e $\mathrm{H}-3 \mathrm{de}$ um anel piridínico do esqueleto azaaporfinoide, ${ }^{19,20}$ e também quatro duplos dupletos duplos compatíveis com um sistema aromático $\mathrm{ABCD}$, sendo um mais desprotegido em $\delta_{\mathrm{H}} 9,13$ (ddd; $J=8,4 ; 1,2$; $0,8 \mathrm{~Hz}$ ) característico do próton $\mathrm{H}-10$ desse tipo de esqueleto, um em $\delta_{\mathrm{H}} 8,26$ (ddd; $J=8,4 ; 1,2 ; 0,8 \mathrm{~Hz}$ ) atribuído ao H-7 e os outros dois em $\delta_{\mathrm{H}} 7,81$ (ddd; $J=8,4 ; 7,2 ; 1,2 \mathrm{~Hz}$ ) e 7,73 (ddd; $J=8,4 ; 7,2$; $1,2 \mathrm{~Hz}$ ) assinalados para $\mathrm{H}-8$ e $\mathrm{H}-9$, respectivamente. Além desses sinais, foram ainda observados dois simpletos, ambos integrando para três prótons, em $\delta_{\mathrm{H}} 4,07$ (s) e $\delta_{\mathrm{H}} 4,04(\mathrm{~s})$, correspondentes a duas metoxilas. No espectro de ${ }^{13} \mathrm{C}\left(\mathrm{CD}_{3} \mathrm{OD}, 100 \mathrm{MHz}\right)$ foram observados dezesseis sinais. Desses, seis denotam ser de carbonos metínicos aromáticos e dois de grupos metoxila, confirmando dessa forma os dados observados no espectro de RMN de ${ }^{1} \mathrm{H}$. Observou-se também um sinal característico de um grupo carbonila e mais sete sinais indicativos de carbonos aromáticos não hidrogenados. A análise desses dados e do espectro de correlações HSQC permitiram atribuir os sinais dos carbonos metínicos em $\delta_{\mathrm{C}}$ 147,3 e 121,8 aos carbonos 
<smiles>COc1c(OC)c2c(C(N)=O)ccnc2c2ccccc12</smiles>

1<smiles></smiles>

$$
\begin{aligned}
& \text { 2: } \mathrm{R}=\mathrm{OCH}_{3} \\
& \text { 3: } \mathrm{R}=\mathrm{H}
\end{aligned}
$$<smiles>[R]c1cnc2c3c(nccc13)-c1ccccc1-2</smiles>

4: $\mathrm{R}=\mathrm{OCH}_{3}$ 5: $\mathrm{R}=\mathrm{H}$<smiles>Cc1cnc2c3c(nccc13)-c1ccccc1C2=O</smiles>

6

Figura 1. Alcaloides isolados de A. dolichocarpa

\begin{tabular}{|c|c|c|c|c|c|}
\hline \multirow{2}{*}{ Posição } & \multicolumn{2}{|c|}{ Dolichocarpina $(\mathbf{1})^{1}$} & \multirow{2}{*}{ Posição } & \multicolumn{2}{|c|}{ 9-metoxieupolauramina $(\mathbf{2})^{2}$} \\
\hline & $\delta_{\mathrm{H}}($ mult,$J)$ & $\delta_{\mathrm{C}}$ & & $\delta_{\mathrm{H}}($ mult,$J$ ) & $\delta_{\mathrm{C}}$ \\
\hline 2 & $8,95(\mathrm{~d} ; 4,8)$ & 147,3 & 2 & $9,13(\mathrm{~d} ; 4,8)$ & 149,9 \\
\hline 3 & $7,64(\mathrm{~d} ; 4,8)$ & 121,8 & 3 & $7,92(\mathrm{~d} ; 4,8)$ & 117,4 \\
\hline $3 a$ & - & 144,4 & $3 a$ & - & $133,1^{*}$ \\
\hline 4 & - & 174,4 & 4 & - & $166,6^{*}$ \\
\hline 5 & - & 143,8 & $5 \mathrm{a}$ & - & $122,8^{*}$ \\
\hline 6 & - & 147,4 & 6 & - & $138,1^{*}$ \\
\hline $6 a$ & - & 132,0 & $6 a$ & - & $126,2^{*}$ \\
\hline 7 & $8,26(\mathrm{ddd} ; 8,4 ; 1,2 ; 0,8)$ & 122,9 & 7 & $8,04(\mathrm{~d} ; 9,2)$ & 124,3 \\
\hline 8 & $7,81(\mathrm{ddd} ; 8,4 ; 7,2 ; 1,2)$ & 130,8 & 8 & $7,33(\mathrm{dd} ; 9,2 ; 2,4)$ & 119,3 \\
\hline 9 & 7,73 (ddd; 8,4; 7,2; 1,2) & 128,0 & 9 & - & $158,5^{*}$ \\
\hline 10 & $9,13(\mathrm{ddd} ; 8,4 ; 1,2 ; 0,8)$ & 125,4 & 10 & $8,44(\mathrm{~d} ; 2,4)$ & 105,2 \\
\hline $10 \mathrm{a}$ & - & 128,9 & $10 \mathrm{a}$ & - & $131,8^{*}$ \\
\hline $10 \mathrm{~b}$ & - & 144,3 & $10 \mathrm{~b}$ & - & $142,3^{*}$ \\
\hline $10 \mathrm{c}$ & - & 122,1 & $10 \mathrm{c}$ & - & $122,9 *$ \\
\hline $\mathrm{H}_{3} \mathrm{CO}-5$ & $4,04(\mathrm{~s})$ & 61,6 & $\mathrm{H}_{3} \mathrm{CO}-6$ & $4,05(\mathrm{~s})$ & 55,8 \\
\hline \multirow[t]{2}{*}{$\mathrm{H}_{3} \mathrm{CO}-6$} & 4,07 (s) & 61,3 & $\mathrm{H}_{3} \mathrm{CO}-9$ & $4,04(\mathrm{~s})$ & 63,6 \\
\hline & & & $\mathrm{H}_{3} \mathrm{C}-\mathrm{N}$ & $3,72(\mathrm{~s})$ & 28,5 \\
\hline
\end{tabular}

Tabela 1. Dados de RMN de ${ }^{1} \mathrm{H}(400 \mathrm{MHz})$ e ${ }^{13} \mathrm{C}(100 \mathrm{MHz})$ dos compostos $\mathbf{1}$ e 2, $\delta$ em ppm, $J$ em Hz

${ }^{1}$ Obtido em $\mathrm{CD}_{3} \mathrm{OD} ;{ }^{2}$ obtido em $\mathrm{CDCl}_{3} ;{ }^{*}$ dados atribuídos por $\mathrm{HMBC}$.

C-2 e C-3, o primeiro mais desprotegido por estar ligado a um heteroátomo, e em $\delta_{\mathrm{C}} 122,9,130,8,128,0$ e 125,4 aos carbonos C-7, C-8, C-9 e C-10, respectivamente. Os demais carbonos foram definidos com auxílio das correlações a longa distância $\left({ }^{2} J \mathrm{e}^{3} J\right)$ observadas no experimento HMBC (Figura 2), que permitiu também confirmar as demais atribuições já sugeridas. A correlação dos sinais em $\delta_{\mathrm{H}} 8,95$ $(\mathrm{H}-2) \operatorname{com} \delta_{\mathrm{C}} 121,8(\mathrm{C}-3), 144,4(\mathrm{C}-3 \mathrm{a}), 144,3(\mathrm{C}-10 \mathrm{~b})$ e em $\delta_{\mathrm{H}} 7,64$ $(\mathrm{H}-3) \operatorname{com} \delta_{\mathrm{C}} 147,3(\mathrm{C}-2), 174,4$ (C-4), 122,1 (C-10c) estabeleceu os valores dos carbonos $\mathrm{C}-3 \mathrm{a}, \mathrm{C}-10 \mathrm{~b}$ e $\mathrm{C}-10 \mathrm{c}$, bem como ratificou a posição da carbonila em C-4. A correlação dos sinais em $\delta_{\mathrm{H}} 7,81$ $(\mathrm{H}-8)$ e $\delta_{\mathrm{H}} 9,13(\mathrm{H}-10)$ com $\delta_{\mathrm{C}} 132,0(\mathrm{C}-6 \mathrm{a})$ e em $\delta_{\mathrm{H}} 8,26(\mathrm{H}-7)$ e $\delta_{\mathrm{H}} 7,73(\mathrm{H}-9) \operatorname{com} \delta_{\mathrm{C}} 128,9$ (C-10a) determinou o deslocamento dos carbonos C-6a e C-10a. Além dessa correlação, o sinal em $\delta_{\mathrm{H}} 8,26$ (H-7), assim como o $\delta_{\mathrm{H}} 4,07\left(\mathrm{H}_{3} \mathrm{CO}-6\right)$, correlacionaram-se com $\delta_{\mathrm{C}} 147,4$ (C-6), permitindo dessa forma atribuir o deslocamento de C-6, substituído, portanto, por um grupo metoxila. A localização da segunda metoxila foi definida por meio da correlação do sinal em $\delta_{\mathrm{H}} 4,04\left(\mathrm{H}_{3} \mathrm{CO}-5\right)$ com o sinal em $\delta_{\mathrm{C}} 143,8(\mathrm{C}-5)$. Para confirmar o grupo $\mathrm{NH}_{2}$ na estrutura química, a amostra foi submetida a análise de $\mathrm{RMN} \mathrm{de}{ }^{1} \mathrm{Hem} \mathrm{CDCl}_{3}$, na qual pôde-se observar dois sinais largos em $\delta_{\mathrm{H}} 5,74$ e 5,87 que desapareceram ao adicionar-se $\mathrm{D}_{2} \mathrm{O}$ (Figuras $9 \mathrm{~S}$ e 10S). Assim, com base nesses dados, foi possível propor para 1 a estrutura mostrada na Figura 1, um novo alcaloide azafenantreno denominado dolichocarpina. Sua análise por $\mathrm{EM}^{\mathrm{n}}$ apresentou os íons indicados na Figura $2 \mathrm{~S}$ e a proposta de fragmentação desses íons pode ser observada na Figura 3. ${ }^{21,22}$

O composto 2 foi isolado como cristais amarelados. O espectro de EMAR-IES mostrou o pico do íon $[\mathrm{M}+\mathrm{H}]^{+} \mathrm{com} \mathrm{m} / \mathrm{z}$ 295, 1078 (calcd. para $\mathrm{C}_{17} \mathrm{H}_{15} \mathrm{~N}_{2} \mathrm{O}_{3}, 295,1077$ ), compatível com a fórmula molecular $\mathrm{C}_{17} \mathrm{H}_{15} \mathrm{~N}_{2} \mathrm{O}_{3}$. Os dados de RMN dessa substância foram comparados com os dados do alcaloide eupolauramina, isolado previamente de A. dolichocarpa e novamente neste trabalho (composto 3), ${ }^{11}$ como também com outros relatados na literatura. ${ }^{20} \mathrm{O}$ espectro de $\mathrm{RMN}$ de ${ }^{1} \mathrm{H}$ $\left(\mathrm{CDCl}_{3}, 400 \mathrm{MHz}\right)$ apresentou semelhança com o da eupolauramina (3), observando-se porém um sinal de grupo metoxila a mais. Além dessa diferença, o espectro revelou a ausência de um sinal de próton aromático e alteração na multiplicidade dos sinais, apresentando um dupleto duplo e dois dupletos no lugar dos quatro duplos dupletos duplos, sugerindo dessa maneira uma substituição no anel D do esqueleto eupolaumarina. Os sinais em $\delta_{\mathrm{H}} 7,33(\mathrm{dd} ; J=9,2 ; 2,4 \mathrm{~Hz}$ ), $\delta_{\mathrm{H}} 8,04(\mathrm{~d} ; J=9,2 \mathrm{~Hz})$ e $\delta_{\mathrm{H}} 8,44(\mathrm{~d} ; J=2,4 \mathrm{~Hz})$ sugerem substituição no C-9, devido à ausência do sinal mais desprotegido na região próxima de 9,0 ppm atribuído ao H-10, que pode ter sofrido um efeito de proteção orto da metoxila na posição vizinha (C-9), podendo-se 


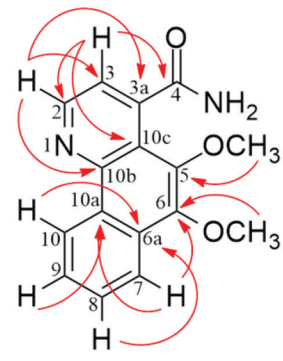

1

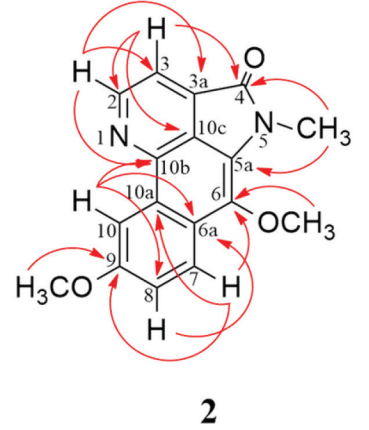

\section{HMBC}

Figura 2. Correlações $H M B C$ de 1 e 2<smiles>COc1c(OC)c2c(C([NH3+])=O)ccnc2c2ccccc12</smiles>

$m / z 283$

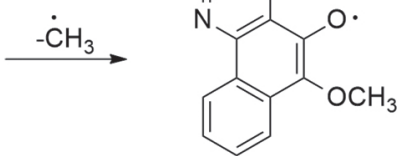

$m / z 268$
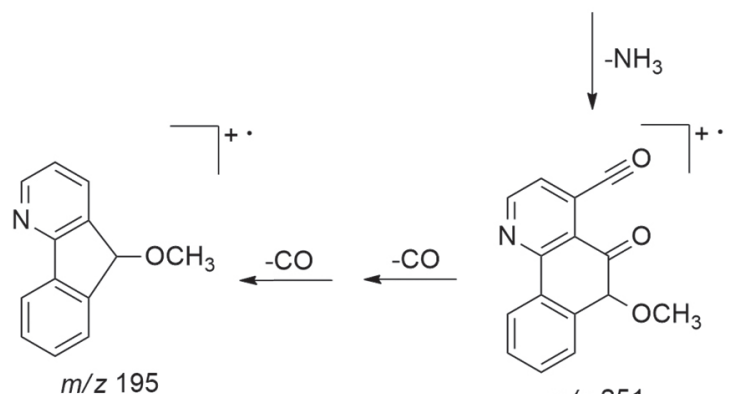

$m / z 251$
Figura 3. Proposta de fragmentação de $E M^{n}$ de 1

então atribuir esses sinais para H-8, H-7 e H-10, respectivamente. No espectro de $\mathrm{RMN}$ de ${ }^{13} \mathrm{C}$ foi possível observar apenas oito sinais. Dentre eles se destaca o sinal de metoxila adicional em $\delta_{\mathrm{C}} 55,8$ e o de um carbono aromático mais protegido em $\delta_{\mathrm{C}} 105,2$, o qual apresentou correlação no espectro HSQC com o sinal em $\delta_{\mathrm{H}} 8,44$, atribuindo-se, por conseguinte, esse sinal ao carbono $\mathrm{C}$-10. Os carbonos que não tiveram seus sinais observados no espectro de $\mathrm{RMN} \mathrm{de}{ }^{13} \mathrm{C}\left(\mathrm{CDCl}_{3}\right.$, $100 \mathrm{MHz}$ ) foram assinalados após análise do espectro de correlações HMBC (Figura 2). Esse experimento permitiu também confirmar a posição da metoxila em C-9 por meio da correlação do sinal em

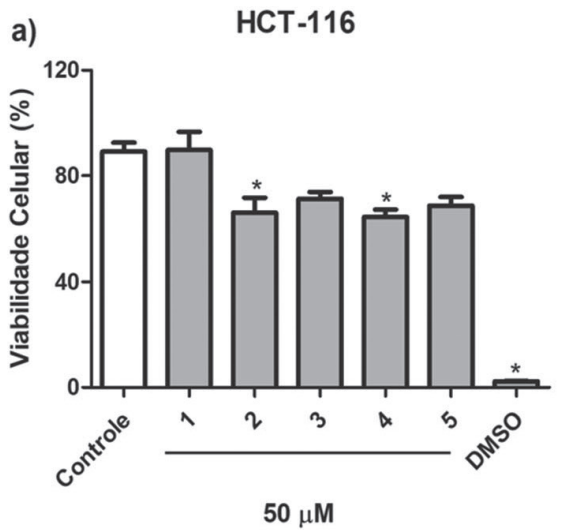

$\delta_{\mathrm{C}} 158,5(\mathrm{C}-9)$ com $\delta_{\mathrm{H}} 4,04\left(\mathrm{H}_{3} \mathrm{CO}-9\right)$ e 8,04 (H-7), e deste último com $\delta_{\mathrm{C}}$ 138,1 (C-6). Assim, o composto 2, pôde ser identificado como um novo alcaloide azafenantreno, denominado 9-metoxieupolauramina.

Composto 3: RMN de ${ }^{1} \mathrm{H}\left(\mathrm{CDCl}_{3}, 400 \mathrm{MHz}\right), \delta$ em ppm (mult.; $J$ em Hz; H): 9,16 (d; 4,8; H-2), 9,01 (ddd; 8,0; 1,6; 0,8; H-10), 8,13 (ddd; 8,0; 1,2; 0,8; H-7), 7,91 (d; 4,8; H-3), 7,73 (ddd; 8,0; 6,8; 1,6; H-8), 7,67 (ddd; 8,0; 6,8; 1,2; H-9), 4,06 (s; H $\mathrm{H}_{3} \mathrm{CO}-6$ ), 3,73 (s; $\mathrm{H}_{3} \mathrm{C}-\mathrm{N}$ ). $\mathrm{RMN}$ de ${ }^{13} \mathrm{C}\left(\mathrm{CDCl}_{3}, 100 \mathrm{MHz}\right), \delta$ em ppm: 166,8 (C-4), 150,1 (C-2), 142,5 (C-10b), 132,6 (C-6a), 129,6 (C-8), 126,8 (C-9), 124,9 (C-5a), 124,4 (C-10), 122,8 (C-7), 117,2 (C-3), 63,5 (H $\left.\mathrm{H}_{3} \mathrm{CO}-6\right), 28,5$ $\left(\mathrm{H}_{3} \mathrm{C}-\mathrm{N}\right)$. EMAR-IES: $\mathrm{m} / z, 265,0979[\mathrm{M}+\mathrm{H}]^{+}$(calcd. para $\mathrm{C}_{16} \mathrm{H}_{13} \mathrm{~N}_{2} \mathrm{O}_{2}$, 265,0972). Dados coerentes com os relatados na literatura para eupolauramina, ${ }^{11}$ já isolado em $A$. dolichocarpa.

Composto 4: RMN de ${ }^{1} \mathrm{H}\left(\mathrm{CDCl}_{3}, 400 \mathrm{MHz}\right), \delta$ em ppm (mult.; $J$ em Hz; H): 8,72 (d; 6,0; H-5), 8,15 (s; H-2), 7,98 (ddd; 6,8; 1,2; 0,8; H-10), 7,91 (ddd; 6,8; 1,2; 0,8; H-7), 7,61 (d; 6,0; H-4), 7,45 (dt; 7,6; 1,2; H-9), 7,40 (dt; 7,6; 1,6; H-8), 4,08 (s; H CO-3). RMN de ${ }^{13} \mathrm{C}\left(\mathrm{CDCl}_{3}, 100 \mathrm{MHz}\right), \delta$ em ppm (alguns dados atribuídos por HMBC): 161,6 (C-6a), 155,0 (C-10b), 150,5 (C-3), 149,2 (C-5), 131,1 (C-9), 129,9 (C-8), 129,4 (C-2), 128,5 (C-3a), 122,6 (C-10), 121,7 (C-7), 113,5 (C-4), 56,4 ( $\left.\mathrm{CH}_{3} \mathrm{O}-3\right)$. EMAR-IES: $m / 2,235,0872$ $[\mathrm{M}+\mathrm{H}]^{+}$(calcd. para $\left.\mathrm{C}_{15} \mathrm{H}_{11} \mathrm{~N}_{2} \mathrm{O}, 235,0866\right)$. Dados compatíveis com os reportados para 3-metoxieupolauridina, ${ }^{23}$ isolado pela primeira vez na família Annonaceae.

Composto 5: RMN de ${ }^{1} \mathrm{H}\left(\mathrm{CDCl}_{3}, 400 \mathrm{MHz}\right), \delta$ em ppm (mult.; $J$ em Hz; H): 8,69 (d; 6,0; H-2/H-5), 7,99 (dd; 8,4; 2,0 H-10/H-7), 7,46 (dd; 8,4; 2,0; H-8/H-9), 7,42 (d; 6,0; H-3/H-4). RMN de ${ }^{13} \mathrm{C}\left(\mathrm{CDCl}_{3}\right.$, $100 \mathrm{MHz}), \delta$ em ppm: 162,7 (C-6a/C-10b), 149,7 (C-2/C-5), 139,6 (C-6b/C-10a), 135,3 (C-3a), 131,4 (C-8/C-9), 122,9 (C-7/C-10), 120,9 (C-10c), 117,6 (C-3/C-4). EMAR-IES: $m / z, 205,0762[\mathrm{M}+\mathrm{H}]^{+}$ (calcd. para $\mathrm{C}_{14} \mathrm{H}_{9} \mathrm{~N}_{2}, 205,0760$ ). Dados semelhantes com os relatados na literatura para eupolauridina, ${ }^{15,24}$ relatado pela primeira vez na espécie A. dolichocarpa.

Composto 6: RMN de ${ }^{1} \mathrm{H}\left(\mathrm{CDCl}_{3}, 400 \mathrm{MHz}\right), \delta$ em ppm (mult.; $J$ em Hz; H): 8,95 (d; 0,8; H-5), 8,89 (d; 6,0; H-2), 8,83 (ddd; 8,0; 1,2; 0,8; H-11), 8,45 (ddd; 8,0; 1,6; 0,8; H-8), 7,81 (ddd; 8,0; 7,6; 1,6; H-10), 7,79 (d; 6,0; H-3), 7,67 (ddd; 8,0; 7,6; 1,2; H-9), 2,76 (d; 0,$8 ; \mathrm{CH}_{3}-4$ ). EMAR-IES: $\mathrm{m} / z$ 247,0875 $[\mathrm{M}+\mathrm{H}]^{+}$(calcd. para $\mathrm{C}_{16} \mathrm{H}_{11} \mathrm{~N}_{2} \mathrm{O}$, 247,0866). Dados compatíveis com os relatados na literatura para 4-metilsampangina, ${ }^{25}$ relatado pela primeira vez como produto natural.

Os compostos 2 e 4 inibiram significativamente a proliferação de células HCT-116 com percentual de inibição de 30,22 $\pm 2,63 \%$ e $32,42 \pm 0,53 \%$, respectivamente (Figura 4). Assim, comparando-se as estruturas químicas de $\mathbf{2}$ com $\mathbf{3}$ e de $\mathbf{4}$ com 5, observou-se nos dois casos uma substituição por um grupo metoxila em $\mathbf{2}$ e $\mathbf{4}$, o que

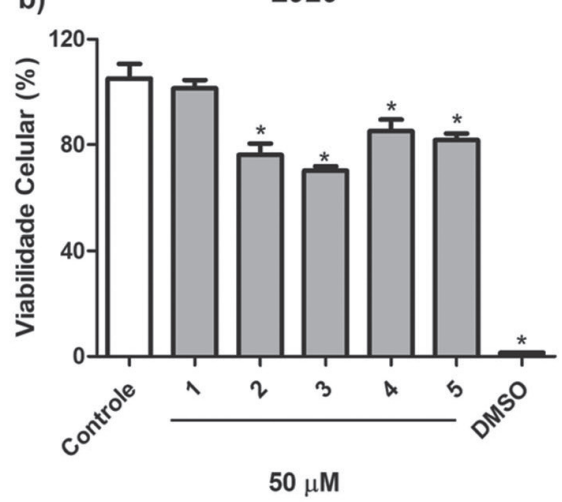

Figura 4. Citotoxicidade dos compostos 1 - 5 em linhagens de células HCT-116 e L929, após 72 h de tratamento. Os valores representam a média \pm erro padrão de quatro replicatas na concentração de $50 \mu \mathrm{mol} L^{-1}$ (*p $<0,05$ comparado ao grupo controle) 
permitiu sugerir que essa substituição aumentou a atividade citotóxica desses compostos para células tumorais da linhagem HCT-116. O estudo de citotoxicidade em linhagem não tumoral de fibroblasto de camundongo L929 mostrou que os compostos $\mathbf{2}$ - $\mathbf{5}$ podem reduzir a proliferação celular, o que indica citotoxicidade.

\section{CONCLUSÃO}

O estudo do extrato etanólico das raízes de A. dolichocarpa levou ao isolamento, por cromatografia líquida de média pressão e cromatografia líquida de alta eficiência, dos novos alcaloides azafenantrenos dolichocarpina (1) e 9-metoxieupolauramina (2), além dos alcaloides eupolauramina (3), já isolado na espécie, 3-metoxieupolauridina (4), novo na família, eupolauramina (5), novo na espécie e 4-metilsampangina (6), relatado pela primeira vez como produto natural. O estudo da atividade citotóxica mostrou que os compostos $\mathbf{2}-\mathbf{5}$ induziram citotoxicidade em linhagem celular não tumoral murina L929 e que, em linhagem tumoral HCT-116, apenas os compostos $\mathbf{2}$ e $\mathbf{4}$ foram capazes de inibir a proliferação celular.

\section{PARTE EXPERIMENTAL}

\section{Procedimentos experimentais gerais}

As separações por cromatografia líquida de média pressão (CLMP) foram feitas utilizando como fase estacionária sílica gel $(40-63 \mu \mathrm{m}$, 230-400 mesh, SiliCycle) e um equipamento Buchi modelo Sepacore flash system X-50. O software desse equipamento permitiu analisar as frações coletadas, auxiliando desse modo no agrupamento das amostras. As frações eluídas foram também analisadas por cromatografia de camada delgada (CCD) utilizando-se placas de alumínio pré-revestidas com sílica gel $\mathrm{F}_{254}$ (SiliCycle), revelando-se com reagente de Dragendorff e exposição à luz UV (254 e $366 \mathrm{~nm})$, e agrupadas de acordo com a similaridade de eluição. A cromatografia líquida de alta eficiência (CLAE) analítica foi realizada no instrumento Shimadzu Prominence equipado com uma bomba de solvente binária LC-20AT, auto-injetor SIL-20A, sistema degaseificador DGU-20A, detector DAD SPD-M20A, sistema de controle CBM-20A e as colunas de fase reversa Kromasil 100-10-C18 (250 mm × 4,6 mm preenchida com partículas de $10 \mu \mathrm{m})$ ou ACE 5 C18 $(250 \mathrm{~mm} \times 4,6 \mathrm{~mm}$ e partículas de $5 \mu \mathrm{m})$. As frações foram purificadas por CLAE na escala semi-preparativa e preparativa. Para a primeira técnica foi utilizado um equipamento Shimadzu composto por bomba LC-10AD vp, válvula solenoide FCV-10AL vp, injetor manual Rheodyne, desgaseificador DGU-14A, detector UV-vis SPD-10A vp, controlador de sistema SLC-10A vp e uma coluna semi-preparativa Venusil XBP C18 (250 mm $\times 10 \mathrm{~mm}$ e partículas de $10 \mu \mathrm{m})$. No caso da escala preparativa a purificação foi realizada em um Shimadzu contendo duas bombas LC-6AD, injetor manual Rheodyne, detector DAD SPD-M10A vp, controlador de sistema SLC-10A vp e coluna preparativa ACE 5 C18 (250 mm × 21,2 mm e partículas de $5 \mu \mathrm{m})$. O fluxo empregado para as purificações foi de $3,5 \mathrm{~mL} \mathrm{~min}{ }^{-1}$ para a escala semipreparativa e $8,0 \mathrm{~mL} \mathrm{~min}{ }^{-1}$ para a preparativa, realizando-se, para ambas, injeções de $100 \mu \mathrm{L}$ de amostra para cada corrida cromatográfica.

Os espectros de massa de alta resolução com ionização por electrospray (EMAR-IES) e de baixa resolução em tandem (MS ${ }^{n}$ ) foram obtidos em equipamentos Bruker modelos micrOTOF II e Ion Trap-amaZonX, respectivamente, os dois operando no modo positivo. Os espectros de RMN de ${ }^{1} \mathrm{H},{ }^{13} \mathrm{C}$ e bidimensionais foram adquiridos no espectrômetro Bruker AVANCE III HD (400 MHz e $100 \mathrm{MHz}$ para ${ }^{1} \mathrm{H}$ e ${ }^{13} \mathrm{C}$, respectivamente) usando $\mathrm{CDCl}_{3}$ e $\mathrm{CD}_{3} \mathrm{OD}$ como solvente e $\mathrm{CHCl}_{3}\left(\delta_{\mathrm{H}} 7,24\right.$ e $\left.\delta_{\mathrm{C}} 77,0\right)$ e $\mathrm{CH}_{3} \mathrm{OH}\left(\delta_{\mathrm{H}} 3,30\right.$ e $\left.\delta_{\mathrm{C}} 49,0\right)$ residual como padrão interno. As análises de infravermelho foram realizadas nos espectrômetros Rayleigh WQF-510 FT-IR e PerkinElmer Frontier FT-IR, com pastilhas de $\mathrm{KBr}$ e número de onda medido em $\mathrm{cm}^{-1}$.

\section{Material vegetal}

As raízes de Anaxagorea dolichocarpa Sprague \& Sandwith foram coletadas em Cruz do Espírito Santo, Paraíba, Brasil em dezembro de 2010. O registro de acesso ao Sistema Nacional de Gestão do Patrimônio Genético e do Conhecimento Tradicional Associado (SisGen) foi obtido sob o número AE4B71A. A identificação botânica foi realizada pela Dr ${ }^{\mathrm{a}}$ Maria de Fátima Agra e uma exsicata encontra-se depositada no Herbário Prof. Lauro Pires Xavier (JPB), no Centro de Ciências Exatas e da Natureza, registrada como AGRA \& GÓES 5543.

\section{Extração e isolamento}

As raízes de A. dolichocarpa foram secas em estufa de ar circulante a $45{ }^{\circ} \mathrm{C}$ por $92 \mathrm{~h}$ e em seguida trituradas produzindo 700,0 g de pó. Esse material foi, então, submetido ao processo de extração por maceração com etanol $95 \%$ por $72 \mathrm{~h}$, em cinco repetições. A solução extrativa obtida foi concentrada em evaporador rotativo a $45^{\circ} \mathrm{C}$, resultando em 77,6 g de extrato etanólico bruto (EEB). Uma alíquota de 76,1 g do EEB foi inicialmente submetida a uma extração com hexano, para separação dos constituintes com baixa polaridade, permanecendo em contato com o solvente, em agitação mecânica, por cerca de 5 minutos. A seguir o material foi filtrado e o resíduo insolúvel pesando $66,4 \mathrm{~g}$ foi submetido ao procedimento de extração ácido-base para alcaloides ${ }^{26}$ fornecendo $2,3 \mathrm{~g}$ de fração alcaloídica (FA). Uma amostra da FA (2,1 g) foi fracionada por CLMP, usando sílica gel previamente tratada com $\mathrm{NaHCO}_{3} 10 \%,{ }^{26}$ e gradientes dos solventes $\mathrm{Hex}-\mathrm{CHCl}_{3}(100: 0 \rightarrow 0: 100), \mathrm{CHCl}_{3}$-AcOEt (100:0 $\rightarrow$ 0:100) e AcOEt:MeOH (100:0 $\rightarrow$ 3:7), resultando em 116 frações de $50 \mathrm{~mL}$ que após análise em CCD foram agrupadas em 20 (FA1-FA20). A fração FA2 (95,0 mg), eluída em $\mathrm{Hex}_{-} \mathrm{CHCl}_{3}$ (8:2), foi submetida a um novo fracionamento por CLMP, com sílica gel tratada com $\mathrm{NaHCO}_{3}$ $10 \%$, e gradiente de $\mathrm{Hex}-\mathrm{CHCl}_{3}(100: 0 \rightarrow 0: 100)$ e $\mathrm{CHCl}_{3}$-AcOEt (100:0 $\rightarrow$ 0:100), fornecendo 9 subfrações (FA2A-FA2I). A subfração FA2D (15,8 mg), obtida em $\mathrm{Hex}-\mathrm{CHCl}_{3}$ (95:5), foi purificada por CLAE semi-preparativa com sistema gradiente de água $(0,1 \%$ de ácido fórmico) e $\mathrm{MeOH}(95: 5 \rightarrow$ 0:100, em 60 minutos) a $254 \mathrm{~nm}$, dando origem ao composto 5 (2,0 mg), eluído em $74 \%$ de $\mathrm{MeOH}$. A subfração FA2E $(23,4 \mathrm{mg})$, também obtida em $\mathrm{Hex}-\mathrm{CHCl}_{3}$ (95:5), foi purificada por CLAE semi-preparativa usando o seguinte gradiente de eluição: solvente $\mathrm{A}$ = água $(0,1 \%$ de ácido fórmico); solvente $\mathrm{B}=\mathrm{MeOH}$; sistema de eluição = 0-40 $\min (20-70 \%$ de B); 40-50 min (70\% de B); e 50-65 min (70-100\% de B); a $254 \mathrm{~nm}$; fornecendo as substâncias 2 (0,6 mg), 3 (4,7 mg) e 4 (1,5 mg), eluídas em $80 \%, 72 \%$ e $70 \%$ de B, respectivamente. A fração FA7 (197,3 mg), eluída em $\mathrm{Hex}_{-} \mathrm{CHCl}_{3}$ (1:9), foi purificada por CLAE preparativa com o gradiente de eluição: solvente $\mathrm{A}=$ água ( $0,1 \%$ de ácido fórmico); solvente $\mathrm{B}=\mathrm{MeOH}$; sistema de eluição = 0-45 $\min (0-65 \%$ de B $)$; 45$55 \mathrm{~min}(65 \%$ de B); e $55-90 \mathrm{~min}(65-100 \%$ de B); a $254 \mathrm{~nm}$; isolando 6 (1,5 mg), obtido em 65\% de B. A fração FA8 (295,5 mg), eluída em $\mathrm{CHCl}_{3}$-AcOEt (9:1), foi separada em 11 frações (FA8A-FA8M) por CLMP, com sílica gel tratada com $\mathrm{NaHCO}_{3} 10 \%$, e gradiente de $\mathrm{Hex}_{-} \mathrm{CHCl}_{3}(100: 0 \rightarrow 0: 100), \mathrm{CHCl}_{3}$-AcOEt $(100: 0 \rightarrow 0: 100) \mathrm{e}$ AcOEt:MeOH (100:0 $\rightarrow$ 3:7). A subfração FA8G (121,4 mg), eluída em $\mathrm{CHCl}_{3}$, foi submetida a CLAE semi-preparativa com o gradiente de eluição: solvente $\mathrm{A}$ = água ( $0,1 \%$ de ácido fórmico); solvente $\mathrm{B}=\mathrm{MeOH}$; sistema de eluição = 0-20 $\min (30-40 \%$ de B); 20-65 min (40\% de B); e 65-100 min (40-100\% de B); a $254 \mathrm{~nm}$; obtendo-se o composto 1 (3,6 mg), obtido em $45 \%$ de B. 


\section{Avaliação da citotoxicidade}

Para a avaliação da citotoxicidade dos compostos $\mathbf{1}$ - $\mathbf{5}$ foram utilizadas as linhagens de células HCT-116 (carcinoma colorretal humano) e L929 (fibroblastos murinos não-tumorais). As células foram cultivadas em meio RPMI 1640 suplementado com $10 \%$ de soro bovino fetal, $100 \mathrm{U} \mathrm{mL}^{-1}$ de penicilina e $100 \mu \mathrm{g} \mathrm{mL}^{-1}$ de estreptomicina a $37^{\circ} \mathrm{C}$ em uma atmosfera umidificada de $5 \% \mathrm{CO}_{2}$. As células foram semeadas em placas de 96 poços em uma densidade de $3 \times 10^{5}$ células/ poço. Após um período de $24 \mathrm{~h}$, as células foram incubadas com os compostos $\mathbf{1}$ - $\mathbf{5}\left(50 \mu \mathrm{mol} \mathrm{L}{ }^{-1}\right)$ dissolvidos em DMSO $(0,4 \%)$, por 72 h. Então, o sobrenadante foi descartado e a solução de MTT [brometo de 3-(4,5-dimetiltiazol-2-il)-2,5-difeniltetrazólio] $\left(5 \mathrm{mg} \mathrm{mL}^{-1}\right)$ foi adicionada e incubada por mais $3 \mathrm{~h}$. O sal de formazam depositado foi dissolvido em dodecil sulfato de sódio (SDS) $(100 \mu \mathrm{L}) .{ }^{27} \mathrm{O}$ controle positivo foi o DMSO (20\%). A densidade ótica foi medida em um leitor de microplacas (Synergy HT, BioTek ${ }^{\circledR}$ ) no comprimento de onda de $570 \mathrm{~nm}$. Os resultados foram expressos como média \pm e.p.m. (erro padrão da média) de quatro replicatas e foram comparados por análise de variância (ANOVA - one way), seguido do pós-teste de Tukey. Os resultados foram considerados significativos quando $\mathrm{p}<0,05$.

\section{MATERIAL SUPLEMENTAR}

Os espectros no IV, de RMN uni e bidimensionais, EMAR-IES e de baixa resolução MS $^{n}$ estão disponíveis em http://quimicanova. sbq.org.br, na forma de arquivo PDF, com acesso livre.

\section{AGRADECIMENTOS}

Os autores agradecem às agências de Coordenação de Aperfeiçoamento de Pessoal de Nível Superior (CAPES) (código de financiamento 001) e ao Conselho Nacional de Desenvolvimento Científico e Tecnológico (CNPq) pelo suporte financeiro e bolsas de pesquisa, ao Laboratório Multiusuário de Caracterização e Análise (LMCA-UFPB) e ao Centro Analítico de Instrumentação da Universidade de São Paulo (Central Analítica IQ-USP) pela aquisição dos espectros, e à Rede Norte-Nordeste de Fitoprodutos (INCT-RENNOFITO) pela colaboração.

\section{REFERÊNCIAS}

1. Guo, X.; Tang, C. C.; Thomas, D. C.; Couvreur, T. L. P.; Saunders, R. M. K.; Sci. Rep. 2017, 7, 1.

2. Couvreur, T. L. P.; Helmstetter, A. J.; Koenen, E. J. M.; Bethune, K.; Brandão, R. D.; Little, S. A.; Sauquet, H.; Erkens, R. H. J.; Front. Plant Sci. 2019, 9, 1941.

3. Lopes, J. C.; Mello-Silva, R.; Rev. Bras. Frutic. 2014, 36, 125.

4. Lobão, A. Q.; de Araujo, D. S. D.; Kurtz, B. C.; Rodriguésia 2005, 56, 85.

5. Lúcio, A. S. S. C.; Almeida, J. R. G. S.; Da-Cunha, E. V. L.; Tavares,
J. F.; Barbosa Filho, J. M. In The Alkaloids: Chemistry and Biology; Knölker, H.-J., ed.; Academic Press: San Diego, 2015, vol. 74, pp. 233-409.

6. González-Esquinca, A. R.; De-La-Cruz-Chacón, I.; Castro-Moreno, M.; Orozco-Castillo, J. A.; Riley-Saldaña, C. A.; Rev. Bras. Frutic. 2014, 36, 01.

7. Teichert, H.; Dötterl, S.; Gottsberger, G.; Plant Syst. Evol. 2011, 291, 25.

8. Maas, P. J. M.; Westra, L. Y. T.; Bot. Jahrb. Syst. 1984, 105, 73.

9. Maas, P. J. M.; Westra, L. Y. T.; Bot. Jahrb. Syst. 1985, 105, 145.

10. Scharaschkin, T.; Doyle, J. A.; Syst. Bot. 2005, 30, 712.

11. Lúcio, A. S. S. C.; Almeida, J. R. G. S.; Barbosa-Filho, J. M.; Pita, J. C. L. R.; Branco, M. V. S. C.; Melo, M. F. F.; Agra, M. F.; Da-Cunha, E. V. L.; Da Silva, M. S.; Tavares, J. F.; Molecules 2011, 16, 7125.

12. Pinheiro, R. S.; Rabelo, S. V.; De Oliveira, A. P.; Guimarães, A. L.; De Moraes-Filho, M. O.; Da Costa, M. P.; Pessoa, C. Ó.; Lúcio, A. S. S. C.; Almeida, J. R. G. S.; Trop. J. Pharm. Res. 2016, 15, 793.

13. Sabphon, C.; Temkitthawon, P.; Ingkaninan, K.; Sawasdee, P.; Nat. Prod. Commun. 2015, 10, 301.

14. de Díaz, A. M. P.; Phytochemistry 1997, 44, 345.

15. Husain, K.; Zakaria, S. M.; Lajis, N. H.; Shaari, K.; Ismail, I. S.; Israf, D. A.; Paetz, C.; Phytochem. Lett. 2012, 5, 788.

16. Almeida, J. R. G. S.; Lúcio, A. S. S. C.; Barbosa-Filho, J. M.; Agra, M. F.; da Silva, M. S.; da Cunha, E. V. L.; Uchoa, D. E. A.; Braz-Filho, R.; Biochem. Syst. Ecol. 2007, 35, 456.

17. Hocquemiller, R.; Rasamizafy, S.; Moretti, C.; Jacquemin, H.; Cavé, A.; Planta Med. 1981, 41, 48.

18. Amaral, R. G.; dos Santos, S. A.; Andrade, L. N.; Severino, P.; Carvalho, A. A.; Clin. Oncol. 2019, 4, 1562.

19. Guinaudeau, H.; Leboeuf, M.; Cavé, A.; J. Nat. Prod. 1994, 57, 1033.

20. Taylor, W.; Aust. J. Chem. 1984, 37, 1095.

21. da Silva, F. M. A.; Koolen, H. H. F.; de Almeida, R. A.; de Souza, A. D. L.; Pinheiro, M. L. B.; Costa, E. V.; Quim. Nova 2012, 35, 944.

22. Stévigny, C.; Jiwan, J. H.; Rozenberg, R.; de Hoffmann, E.; QuetinLeclercq, J.; Rapid Commun. Mass Spectrom. 2004, 18, 523.

23. Carroll, A.; Taylor, W.; Aust. J. Chem. 1991, 44, 1615.

24. Hang, N. T. M.; Oanh, N. T. T.; Hue, C. T.; Tung, T. H.; Thoa, H. T.; Thanh, L. N.; Giap, T. H.; Dung, N. A.; Van Hung, N.; Van Minh, C.; Vietnam J. Chem. 2015, 53, 73 .

25. Hufford, C. D.; Zjawiony, J. K.; Srivastava, A. R.; Clark, A. M.; Heterocycles 1994, 39, 779.

26. Costa, E. V.; Pinheiro, M. L. B.; Xavier, C. M.; Silva, J. R. A.; Amaral, A. C. F.; Souza, A. D. L.; Barison, A.; Campos, F. R.; Ferreira, A. G.; Machado, G. M. C.; Leon, L. L. P.; J. Nat. Prod. 2006, 69, 292.

27. Mosmann, T.; J. Immunol. Methods 1983, 65, 55. 